STRUCTURAL SCIENCE CRYSTAL ENGINEERING MATERIALS

ISSN 2052-5206

\section{A priori checking of the light-response and data quality before extended data collection in pump- probe photocrystallography experiments}

\author{
Philip Coppens,* Anna Makal,‡ Bertrand Fournier,§ Katarzyna N. Jarzembska, \\ Radosław Kamiński, $\uparrow$ Krishnayan Basuroy and Elzbieta Trzopł‡
}

Department of Chemistry, University at Buffalo, The State University of New York, Buffalo, NY 14260-3000, USA. *Correspondence e-mail: coppens@buffalo.edu

In picosecond and slower pump-probe diffraction experiments, collection of response-ratio correlation sets prior to full data collection provides an invaluable confirmation of the existence of a light-induced signal prior to full data collection. If a response to light exposure is observed, the quality of the data being collected can be assessed. A number of such correlation plots both for synchrotron and in-house pump-probe data collection are presented.

\section{Introduction}

The Laser-Pump/X-ray probe technique has undergone rapid development in recent years. Much of its current development is taking place at X-ray Free Electron (XFEL) sources using the diffract-and-destroy technique, as illustrated in Kupitz et al. (2014), Schmidt et al. (2015), Tenboer et al. (2014), Marinelli et al. (2015), Hunter et al. (2014) and many other references. However, there is still the need for experiments at picosecond and longer time resolution. They can be performed at synchrotron and more conventional sources in which the sample can survive the stream of X-ray pulses (Neutze \& Moffat, 2012; Lima et al., 2011; Coppens \& Fournier, 2015). This allows direct measurement of the effect of the laser exposure on the diffraction intensities collected on the same crystalline sample, which eliminates systematic errors in the subsequent analysis.

\section{The Ratio method}

In the measurement of the response of the diffraction pattern to photoinduced excitation of the component species in the crystals, the Ratio method has several advantages (Coppens et al., 2009). It implies rapid alternate collection of individual light-ON and light-OFF reflection frames, and thus eliminates the need for relative scaling of different frames as well as errors in all but short-term variations in the X-ray source intensity. Moreover, the subsequent analysis of the laserinduced changes is based on the $\mathrm{ON}-\mathrm{OFF}$ ratios rather than on the individual reflection intensities. Therefore, it removes the effect of anisotropic absorption which would have to be corrected if different crystals were used in the laser-ON and laser-OFF experiments.

When the Laue technique is used at synchrotron sources it also eliminates the need for establishing the spectral distribution of the source, as discussed in detail in a recent publication (Fournier et al., 2016). 


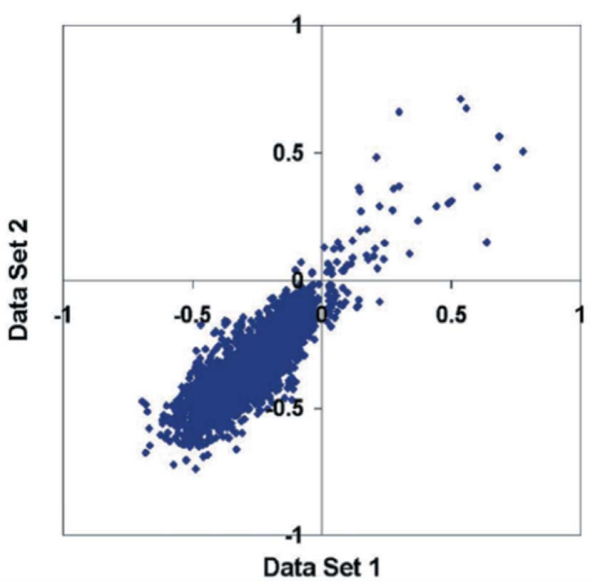

Figure 1

Correlation between two sets of response ratios for $\left[\mathrm{Rh}_{2}(\text { dimen })_{4}\right]\left(\mathrm{PF}_{6}\right)_{2}$, cut-off: reflections with $\sigma(\eta)|\eta| />1$ eliminated; $\eta=R-1$.

Availability of a first set of ON-OFF ratios allows a rapid preliminary analysis to check the presence of light-induced changes and to estimate the data quality. The correlation between the signals in small preliminary data sets on the same crystal either at identical angular setting and laser power or at different laser power provides a rapid test of whether collection of full data sets is warranted. The effect of laser illumination is twofold, an induced structural change will lead to both intensity increases and decreases, the latter because a partial conversion if randomly distributed in the crystal corresponds to a disordered arrangement, and because the laser exposure even though pulsed, invariably causes a temperature increase in the crystal, accounted for in the subsequent refinement by a temperature scale factor. As described in an earlier publication (Vorontsov \& Coppens, 2005) two models can be distinguished. If the excited species are randomly distributed (RD) in the crystal, the coherent diffraction pattern reflects the space-averaged structure, whereas the effect of the photo-induced disorder is reflected in the diffuse scattering pattern. The cluster formation $(\mathrm{CF})$
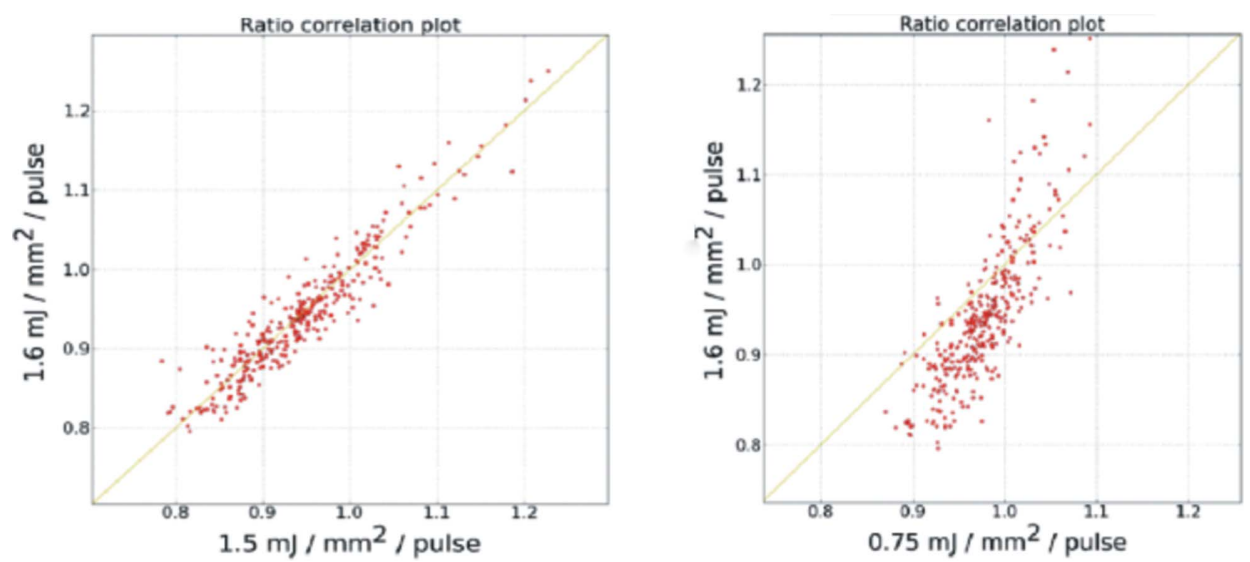

Figure 2

Correlation plots between the ratios of $10^{\circ}$-step data sets on $\mathrm{Cu}^{\mathrm{I}}\left[\left(1,10\right.\right.$-phenanthroline- $\left.N, N^{\prime}\right)$ bis(triphenylphosphine)] at different laser exposures collected at $90 \mathrm{~K}$. The yellow lines here and in the following figures have a $45^{\circ}$ slope. Only data observed on all 10 frames collected at each $\phi$-setting were included. model assumes that the excited molecules cluster to form domains of a new phase with dimensions larger than the X-ray coherence length. This is similar to a photo-induced phase transition, which may lead to the occurrence of a second diffraction pattern or, if the two phases are alike, to a superposition of the reflections of the two phases. A test calculation for the RD model and the CF models shows that the two are well distinguishable, and that in the common RD model the contribution of the disorder leads to a decrease in the ratios of about $20 \%$ for $12 \%$ conversion, which is a higher conversion percentage than achieved in the experiments discussed below, for which the RD model is appropriate.

\section{Examples of response-ratio correlation plots measured prior to full data collection}

1. $\left.\mathrm{Rh}_{2}(\text { dimen })_{4}\right]^{2+}($ dimen $=1,8$-diisocyanomethane $)$ : The first response-ratio correlation plot was published in 2005 (Fig. 1), using monochromatic data collected at beamline 15-ID at the Advanced Photon Source (Coppens et al., 2004, 2005). The experiment, conducted at $17 \mathrm{~K}$, showed a very large contraction of the $\mathrm{Rh}-\mathrm{Rh}$ distance of 0.86 (5) $\AA$, by far the largest structural change observed by atomic resolution time-resolved diffraction experiments on transition metal complexes. Notwithstanding the very low temperature, a very large tail of ratios smaller than one is noticeable, suggesting a large contribution of high-order reflections and a significant temperature increase on laser exposure.

2. $\mathrm{BF}_{4}$ salt of $\mathrm{Cu}^{\mathrm{I}}\left[\left(1,10\right.\right.$-phenanthroline- $\left.N, N^{\prime}\right)$ bis(triphenylphosphine)]: The excited state structure of this complex was determined at both 90 and $180 \mathrm{~K}$ by single-pulse Laue diffraction at BioCARS beamline 14-ID at APS (Makal et al., 2012). The two molecules in the unit cell show different distortion indicative of the effect of the surrounding crystal environment. Ratio correlation plots based on small $90 \mathrm{~K}$ data sets collected at different laser power over a $10^{\circ} \phi$-rotation range are shown in Fig. 2. $\Delta \phi$ step values of 1 and $2^{\circ}$ were used. Laser-OFF and Laser-ON frames were collected in immediate succession to minimize the effect of long-range fluctuations in the beam's position or intensity. The ON/OFF pump-probe cycle was repeated ten times for each frame to allow subsequent statistical background estimation and filtering of the intensities. The first plot (Fig. 2a) shows data collected at almost the same laser power, whereas the second one (Fig. 2b) shows the effect of the increased laser power, as the correlation line deviates from the $45^{\circ}$ equal power diagonal. In both cases the effect of the temperature increase is evident in both data sets, as confirmed by the photo-Wilson plots and the subsequent refinement of a 


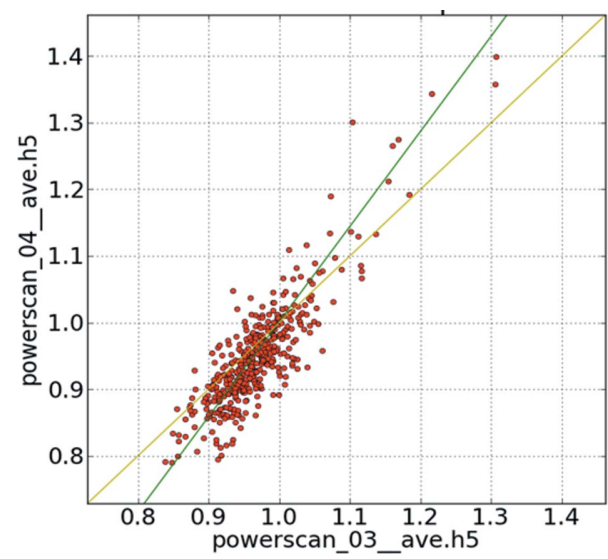

Figure 3

Correlation plot for sets with different laser power on $\mathrm{Ag}_{2} \mathrm{Cu}_{2} \mathrm{I}_{4}$. See text for details.

temperature scale factor (Makal et al., 2012).

3. A coinage metal complex: An example of a correlation plot for the Laue TR experiments on crystals of the tetranuclear coinage metal complex $\mathrm{Ag}_{2} \mathrm{Cu}_{2} L_{4}$ ( $L=2$-diphenylphosphino-3-methylindole ligand) is shown in Fig. 3 . There is about a $10 \%$ difference between the laser power applied in scan $4\left(0.50 \mathrm{~mJ} \mathrm{~mm}^{-2} ; 23.6 \mu \mathrm{J}\right.$ per pulse $)$ and that in scan 3 $\left(0.45 \mathrm{~mJ} \mathrm{~mm}^{-2} ; 25.9 \mu \mathrm{J}\right.$ per pulse $)$, which is clearly seen in the deviation of the slope of the correlation plot from $45^{\circ}$.

4. In-house pump-probe diffraction: In a series of experiments, we have developed the technique of in-house pumpprobe diffraction in order to make the method more generally accessible. The method involves installation of a rotating-disk chopper in the X-ray beam and the use of a delay generator to synchronize the laser and X-ray pulses (Kamiński et al., 2014). In a recent experiment the method was applied to the highly luminescent fully organic bromine compound 1,4-dibromo2,5-bis(octyloxy)benzene $\left(\mathrm{PhBr}_{2} \mathrm{C}_{8}\right)$ (Shi et al., 2016). A response ratio correlation plot between two small data sets on the same crystal is shown in Fig. 4. In this case only reflections with $|\sigma(\eta) /| \eta \mid<0.5$ were included to eliminate less reliable response ratios. The resulting correlation is evident. Subse-

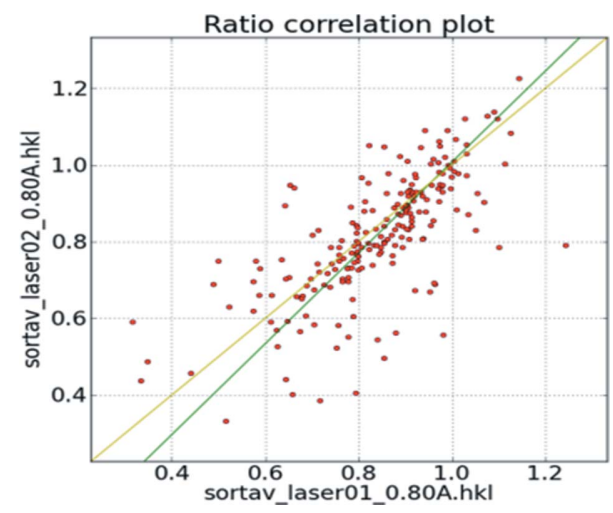

Figure 4

In-house TR response ratios on a crystal of 1,4-dibromo-2,5-bis(hexyloxy)benzene $\left(\mathrm{PhBr}_{2} \mathrm{C}_{8}\right)$. Same laser powers for both data sets. Cut off: $\sigma(\eta) /|\eta| />0.5$ eliminated.

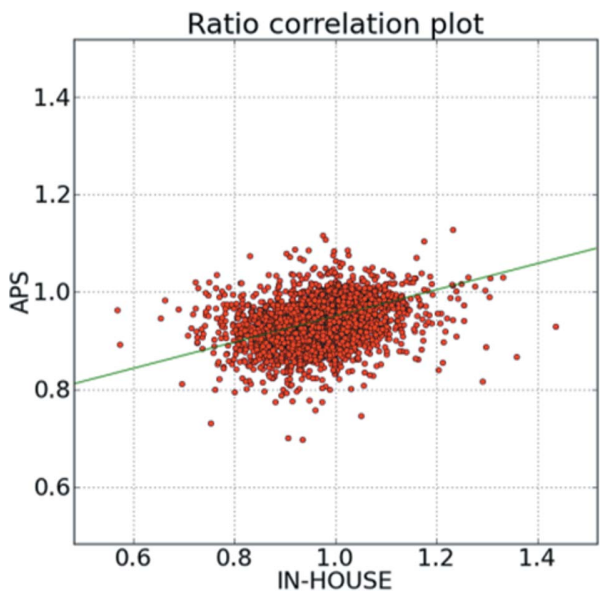

Figure 5

Comparison of synchrotron and in-house data. $7 \mathrm{~ns}$ laser pump pulse delay, Nd-YAG laser, $355 \mathrm{~nm}$. APS: $\sim 80 \mathrm{ps}$ X-ray probe pulse $5.6 \mathrm{~ns}$ pump/probe delay. In house: $30 \mu \mathrm{s}$ X-ray probe pulse $(20.5 \mathrm{kHz})$.

quent analysis of extended data sets led to highly reproducible photodifference maps.

In our earlier work on the in-house data on $\mathrm{Cu}(\mathrm{dmp})$ (dppe) $\cdot \mathrm{PF}_{6}[\mathrm{dmp}=2$,9-dimethyl-1,10-phenanthroline; dppe = 1,2-bis(diphenylphosphino)ethane], response ratios were compared with those collected with the Laue technique at BioCARS beamline 14-ID at APS. Although data collection parameters were very different, the ratio correlation plot shows reasonable agreement between in-house and synchrotron data as shown in Fig. 5 (Trzop et al., 2014).

\section{Response-ratio correlation plots after data processing}

Response-ratio correlation plots can be constructed after processing the data over individual sets. However, as in the case of pre-processing correlation plots discussed above, they are affected when different laser powers are used for different sets, as is often the case. An example is such a plot for the Laue

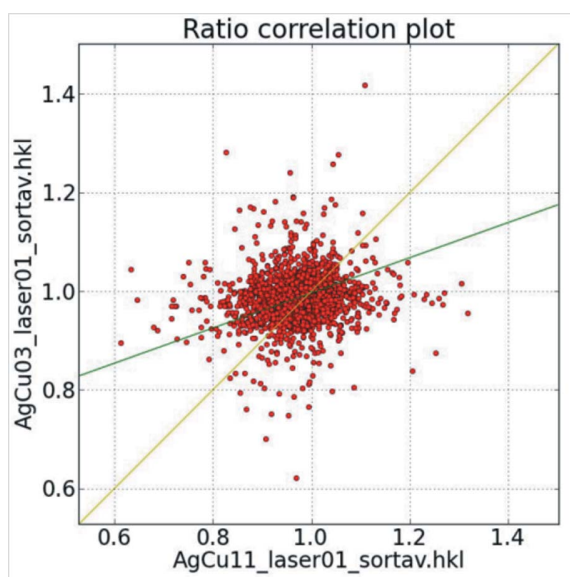

Figure 6

Example of a ratio correlation plot for $\mathrm{Ag}_{2} \mathrm{Cu}_{2} L_{4}$ drawn after processing individual data sets. Laser powers, set $3: 40 \mathrm{~mJ} \mathrm{~mm}^{-2}$, set 11: $0.25 \mathrm{~mJ} \mathrm{~mm}^{-2}$. 
TR experiments on crystals of the tetranuclear coinage metal $\mathrm{Ag}_{2} \mathrm{Cu}_{2} L_{4}$ ( $L=2$-diphenylphosphino-3-methylindole ligand), which shows a pronounced shortening of the $\mathrm{Ag}-\mathrm{Cu}$ distances in $\mathrm{Ag}_{2} \mathrm{Cu}_{2} L_{4}$ on excitation (Jarzembska et al., 2014). Data sets 3 and 11 (referred to as sets 1 and 3 in the supplementary material of the reference) were collected at different laser powers of 0.40 and $0.25 \mathrm{~mJ} \mathrm{~mm}^{-2}$, respectively. As shown in Fig. 6 the higher laser power leads to a larger number of positives above the $y=1$ line compared with the number of positive ratios larger than 1 for set 11 (3) along the $x$-axis. The agreement may also be affected by other factors. Radiation damage due to laser exposure may influence the averages calculated after longer data collection runs. Similar results are obtained for the other data sets. Such differences are properly taken into account in the program LASER (Vorontsov et al., 2010), which allows refinement of up to six individual data sets, each with its own data and temperature scale factors, or in a global refinement of the merged data sets, as described elsewhere (Fournier et al., 2016). The results of the two methods are generally in good agreement.

\section{Conclusions}

We conclude that the collection of response-ratio correlation sets in picosecond and slower pump-probe diffraction experiments prior to full data collection provides an invaluable confirmation of the existence of a light-induced signal and, if a response is observed, the quality of the data being collected. It should be part of any pump-probe data collection project.

\section{Acknowledgements}

Support of this work by the National Science Foundation (CHE-1213223) is gratefully acknowledged. ChemMatCARS, Sector 15, APS, is principally supported by the NSF/DOE under grant No. NSF/CHE-1346572. Use of the BioCARS Sector 14 is supported by the National Institutes of Health, National Center for Research Resources, under grant No. RR007707. The Advanced Photon Source is funded by the US Department of Energy, Office of Basic Energy Sciences, under contract No. W-31-109-ENG-38. KNJ thanks the Polish Ministry of Science and Higher Education for financial support within the Mobility Plus program and the Foundation for Polish Science for financial support within the START program.

\section{References}

Coppens, P. \& Fournier, B. (2015). J. Synchrotron Rad. 22, 280-287. Coppens, P., Gerlits, O., Vorontsov, I. I., Kovalevsky, A. Y., Chen, Y.-S., Graber, T., Gembicky, M. \& Novozhilova, I. V. (2004). Chem. Commun. pp. 2144-2145.

Coppens, P., Pitak, M., Gembicky, M., Messerschmidt, M., Scheins, S., Benedict, J., Adachi, S., Sato, T., Nozawa, S., Ichiyanagi, K., Chollet, M. \& Koshihara, S. (2009). J. Synchrotron Rad. 16, 226-230.

Coppens, P., Vorontsov, I. I., Graber, T., Gembicky, M. \& Kovalevsky, A. Y. (2005). Acta Cryst. A61, 162-172.

Fournier, B., Sokolow, J. \& Coppens, P. (2016). Acta Cryst. A72, 250260.

Hunter, M. S., Segelke, B., Messerschmidt, M., Williams, G. J., Zatsepin, N. A., Barty, A., Benner, W. H., Carlson, D. B., Coleman, M., Graf, A., Hau-Riege, S. P., Pardini, T., Seibert, M. M., Evans, J., Boutet, S. \& Frank, M. (2014). Sci. Rep. 4, 6026.

Jarzembska, K., Kamiński, R., Fournier, B., Trzop, E., Sokolow, J., Henning, R., Chen, Y. \& Coppens, P. (2014). Inorg. Chem. 53, 10594-10601.

Kamiński, R., Nottingham, G. \& Coppens, P. (2014). J. Appl. Cryst. 47, $1765-1768$.

Kupitz, C. et al. (2014). Nature, 513, 261-265.

Lima, F. A., Milne, C. J., Amarasinghe, D. C. V., Rittmann-Frank, M. H., van der Veen, R. M., Reinhard, M., Pham, V., Karlsson, S., Johnson, S. L., Grolimund, D., Borca, C., Huthwelker, T., Janousch, M., van Mourik, F., Abela, R. \& Chergui, M. (2011). Rev. Sci. Instrum. 82, 063111.

Makal, A., Benedict, J., Trzop, E., Sokolow, J., Fournier, B., Chen, Y., Kalinowski, J. A., Graber, T., Henning, R. \& Coppens, P. (2012). J. Phys. Chem. A, 116, 3359-3365.

Marinelli, A., Ratner, D., Lutman, A. A., Turner, J., Welch, J., Decker, F. J., Loos, H., Behrens, C., Gilevich, S., Miahnahri, A. A., Vetter, S., Maxwell, T. J., Ding, Y., Coffee, R., Wakatsuki, S. \& Huang, Z. (2015). Nat. Commun. 6, 6369.

Neutze, R. \& Moffat, K. (2012). Curr. Opin. Struct. Biol. 22, 651-659.

Schmidt, M., Pande, K., Basu, S. \& Tenboer, J. (2015). Struct. Dyn. 2 , 041708.

Shi, H. F., An, Z. F., Li, P. Z., Yin, J., Xing, G. C., He, T. C., Chen, H. Z., Wang, J. G., Sun, H. D., Huang, W. \& Zhao, Y. L. (2016). Cryst. Growth Des. 16, 808-813.

Tenboer, J. et al. (2014). Science, 346, 1242-1246.

Trzop, E., Fournier, B., Jarzembska, K., Sokolow, J., Kaminski, R., Benedict, J., Chen, Y., Henning, R. \& Coppens, P. (2014). Acta Cryst. A70, C776.

Vorontsov, I. I. \& Coppens, P. (2005). J. Synchrotron Rad. 12, 488-493.

Vorontsov, I., Pillet, S., Kamiński, R., Schmøkel, M. S. \& Coppens, P. (2010). J. Appl. Cryst. 43, 1129-1130. 